\title{
Pareto-optimal algorithm in bilateral automated negotiation
}

\begin{abstract}
In this paper we present a Pareto-optimal algorithm in bilateral automated negotiation where the negotiation is modeled by "split the pie" game and alternating-offer protocol. Paretooptimality is the seminal condition in the bargaining problem which leads autonomous agents to the Nash-equilibrium. Generating Pareto-optimal offer in multi-issue bargaining is a computationally complex problem, specially, when autonomous agents have incomplete information about deadline, outside options and the opponent's preferences. Unfortunately, yet to date, there is no articulation that clearly describes an algorithm to generate offer in multi-issue negotiation with perfect information. To this end, we present the maximum greedy trade-offs (MGT) algorithm that generate offers at any aspiration-level in $\mathrm{O}(\mathrm{n})$ with assuming that the order of greedy choices is given, otherwise the complexity will be $\mathrm{O}(\mathrm{n} \mathrm{bg}$ n). We also provide analytical proof for the correctness of the maximum greedy trade-offs algorithm.
\end{abstract}

Keyword: Algorithm; Bilateral negotation; Multi issues; Pareto-optimal 